\title{
Vorwort
}

\section{B. Jakoby}

Online publiziert am 20. Mai 2020

(c) Springer-Verlag GmbH Austria, ein Teil von Springer Nature 2020

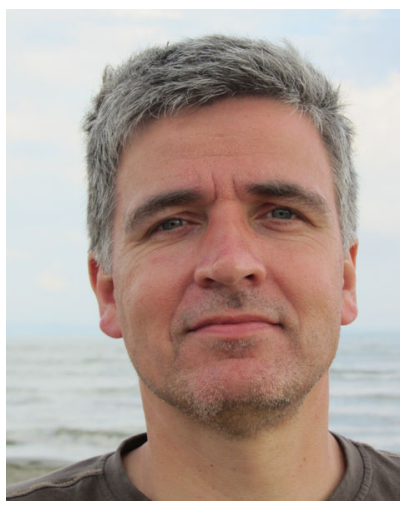

Univ.-Prof. Dr. Bernhard Jakoby
Die Mikrosystemtechnik ist mittlerweile eine seit Jahrzehnten auch in der industriellen Produktion etablierte Technologie. Historisch hat sich die Mikrosystemtechnik auf Basis der Mikroelektronik entwickelt, indem zunächst mechanische Komponenten auf Siliziumchips integriert wurden, um beispielsweise Inertialsensoren (Beschleunigungssensoren, Drehratesensoren etc.) oder Drucksensoren zu realisieren.

Seit geraumer Zeit werden solche Sensoren beispielsweise im Automobil eingesetzt. Wie auch die Mikroelektronik beruht der Erfolg dieser Silizium-basierten Mikrosystemtechnik auf Massenproduktion, da die Fixkosten relativ hoch sind. Mittlerweile hat sich diese Technologie aber weiterentwickelt und viele alternative Fertigungsmöglichkeiten, sei es Nanotechnologie, polymerbasierte Technologien oder Präzisionsmechanik, ergänzen den Werkzeugkasten der Mikrosystemtechnik. Auch das ursprünglich anvisierte Konzept monolithisch integrierter Elektronik und Sensorik hat sich gewandelt, viele der genannten Technologien erlauben gar nicht die Implementierung elektronischer Schaltkreise und dienen ausschließlich zur Realisierung thermischer, fluidischer oder mechanischer "Transducer", also Sensoren oder Aktoren. Die Möglichkeiten der Physik auf der Mikroskala werden etwa bei sogenannten Lab-on-a-chip-Systemen genützt - hierbei werden chemische (oder medizinische oder biologische) Experimente und Untersuchungen mit kleinen Probemengen auf fluidischen Chips (die in der Regel nicht aus Silizium sind) durchgeführt. Sensoren lassen sich oft in diese Chips integrieren, vielfach werden diese Chips aber auch mit großen Analysegeräten kombiniert, sodass diese Lab-on-a-chip-Systeme eher "chips in a Lab" entsprechen.
Die Möglichkeiten sind vielfältig. Entsprechend breit sind auch die Beiträge in dieser Ausgabe gestreut. Sie alle stammen jedoch von Forscherlnnen, die maßgeblich zur österreichischen Sensor-/ Mikrosystemtechnik-/Mikrofluidik-Szene beitragen. Österreich spielt hier international gesehen eine bedeutende Rolle. So kamen etwa bei der letzten IEEE-Sensors-Konferenz in Montreal 20 der insgesamt 471 Beiträge aus Österreich. Das waren mehr als aus TechnologieNationen wie Taiwan oder Italien; die großen europäischen Länder Deutschland und Frankreich waren mit 51 bzw. 34 Beiträgen vertreten. In Österreich ist die Community vor allem in der OVEGesellschaft für Mikroelektronische Systeme und der vom OVE mitgetragenen Gesellschaft für Mess-, Automatisierungs- und Robotertechnik verankert.

Im vorliegenden Heft finden Sie vier Beiträge, die Ihnen einen Einblick in aktuelle Arbeiten und Themengebiete österreichischer Forschungsgruppen in Wien und Linz geben. Zwei Beiträge der TU Wien und der Johannes Kepler Universität Linz (JKU) beschäftigen sich mit Lab-on-a-chip-Systemen - einmal mit der konkreten Betrachtung der Einzelzellanalyse und in einem zweiten Beitrag mit Überlegungen zum Systementwurf. Ein weiterer Beitrag der TU Wien widmet sich aktuellen Untersuchungen zu einer im MEMS-Bereich wesentlichen Materialklasse - den piezoelektrischen Dünnfilmen; im konkreten Fall zur Realisierung miniaturisierter Resonatoren. Ein Beitrag der JKU beleuchtet schließlich eine Lowcost-Fertigungstechnologie, die - alternativ zu klassischen MEMSVerfahren - zur Realisierung von mikrofluidischen Komponenten verwendet werden kann.

Ich wünsche Ihnen eine interessante Lektüre - vielleicht regt Sie ja der eine oder andere Artikel an, sich mit Forscherlnnen dieses interdisziplinären Gebiets in Verbindung zu setzen, um ein Problem in Ihrem eigenen Bereich anzugehen!

Hinweis des Verlags Der Verlag bleibt in Hinblick auf geografische Zuordnungen und Gebietsbezeichnungen in veröffentlichten Karten und Institutsadressen neutral.
Jakoby, Bernhard, Institut für Mikroelektronik und Mikrosensorik, Johannes Keple Universität Linz, Linz, Österreich (E-Mail: bernhard.jakoby@jku.at) 